Special Issue: Cutting-Edge Research on Intestinal Immunity and Inflammation Mini Review

\title{
Epithelial regeneration by transplantation of cultured intestinal stem cells
}

\section{Tetsuya Nakamura}

Department of Advanced Therapeutics for GI Diseases, Tokyo Medical and Dental University, Tokyo, Japan

Research on intestinal epithelial stem cells has flourished in the last few years since their specific markers were identified. However, to exploit the potentials of those adult stem cells as a source for regenerative medicine, validation of the tissue regeneration capability of intestinal stem cells would be essential. We have recently shown that, by employing murine models of transplantation, cultured intestinal epithelial cells from the adult colon, fetal small intestine (SI), and adult SI are able to regenerate epithelia in vivo, preserving their stem cell properties. These data provide the evidence that multiple types of intestinal cells could be the source for the stem cell therapy for intestinal diseases in humans.

Rec.9/7/2014, Acc.9/16/2014, pp19-22

Correspondence should be addressed to:

Tetsuya Nakamura, Department of Advanced Therapeutics for GI Diseases, Tokyo Medical and Dental University, 1-5-45 Yushima, Bunkyo-ku, Tokyo 113-8519, Japan. Phone: +81-3-5803-5877, Fax: +81-3-5803-0268, E-mail: nakamura.gast@tmd.ac.jp

Key words intestinal stem cells, regenerative medicine, stem cell culture, transplantation

\section{Introduction}

With the advancement of stem cell research, there has emerged a growing interest in the use of various stem cells for the replacement therapy for human diseases. Given the long-standing success of hematopoietic stem cell transplantation in clinic, such an approach seems to be promising in therapeutic applications for many other clinical settings. Research on the gastrointestinal (GI) epithelial stem cells has flourished in the last several years. Identification of their specific molecular markers has greatly facilitated characterization of these GI stem cell populations ${ }^{1)}$. In addi- tion, long-awaited technologies that allow for expansion of adult intestinal stem cells in vitro have recently become available ${ }^{2-5)}$. Future progress in this research field would be directed toward the use of expanded intestinal stem cells in culture for the therapy of GI diseases in humans.

\section{Intestinal epithelial stem cells}

Intestinal epithelial tissues are unique in that they continue to self-renew throughout our lifetime. The homeostasis with this perpetual, rapid cellular turnover is governed by the tissue-resident adult stem cells sitting near the base of 
glandular structures called crypts $^{6-8)}$. The stem cells divide continuously, generating more committed precursor cells called transit-amplifying (TA) cells that occupy the lower part of the crypt. As cells differentiate into mature cell lineages, they migrate upward and then are exfoliated into the lumen, whereas Paneth cells, which are unique only to the small intestine (SI), migrate down to the crypt base.

The repair response to epithelial injuries caused by a variety of insults also involves the stem cell-mediated tissue regeneration ${ }^{9,10)}$. Acute phase response to the loss of epithelial linings induces the rapid process called epithelial restitution. In this phase, epithelial cells that survive at the edge of the wound migrate and cover the denuded region to restore the structural integrity of the epithelium. Following this, as the flat epithelial lining dramatically changes its shape and reforms crypt-villus structures in the SI or crypts in the colon, stem cell-based regulations of cellular proliferation and differentiation coordinate morphological and functional repair of injured epithelia.

Several regulatory signals such as the Wnt, bone morphogenic protein (BMP), and Notch pathways play important roles in both steady-state maintenance and repair response upon injury ${ }^{11,12)}$. Among these, the Wnt pathway is regarded as a key regulator for proliferation of the stem cells in intestinal crypts. A large body of evidence shows that intracellular signaling events triggered by the binding of Wnt proteins to their cell surface receptors are essential to maintain the crypt cell population in a proliferative state ${ }^{13-15)}$. In line with this, one of the Wnt target genes, a G proteincoupled receptor Lgr5, has been identified as a specific molecular marker of the stem cells of the SI and colon ${ }^{1)}$. Although the presence of other populations of intestinal stem cells has been proposed thereafter ${ }^{16-18)}$, several lines of evidence, such as the data obtained by genetic lineage tracing experiments, show that the Lgr5+ cells represent long-lived, actively-dividing multipotent stem cells in both the SI and colonic epithelia ${ }^{1)}$.

\section{Culturing intestinal stem cells}

Following identification of stem cell populations, another important advance has been made in intestinal stem cell research. Sato et al. developed an elegant method to culture intestinal stem cells under specific conditions ${ }^{3)}$. When isolated and three-dimensionally embedded, SI crypts were shown to grow as organoids that resemble the physiological epithelial architecture with properly situated stem cells. Importantly, this condition requires Rspo1
(Wnt agonist), Noggin (BMP inhibitor) and EGF, but no support of non-epithelial cells. This indicates that, with the appropriately supplied factors, intestinal stem cells can be maintained even in the absence of non-epithelial cell types that had been thought to be critical components of stem cell niche in vivo. The culture can be maintained for a long period of time, with structures containing Lgr5+ stem cells as well as all types of terminally differentiated cells. The group has further reported similar three-dimensional culture technologies for gastric and colonic epithelial stem cells $^{2,5,19)}$. We have also developed an in vivo culture method for colonic stem cells ${ }^{20}$. In our protocol, isolated colonic crypts are three-dimensionally placed in the collagen gel, and cultured in serum-free defined medium containing Wnt3, Rspo1, Noggin, EGF, HGF, and bovine serum albumin (BSA). Under this condition, the colonic cells form round cystic structures that contain Lgr5+ stem cells as well as terminally differentiated cells of colonic phenotype ${ }^{20)}$. It is of note that these newly introduced intestinal epithelial culture methods, including the one that we developed, allow Lgr5+ putative stem cells to increase in number ex vivo.

\section{Transplantation of cultured stem cell- containing epithelial organoids}

Now that seemingly infinite numbers of Lgr5+ stem cells become available by efficient in vitro culture technologies, the next step toward their use for regenerative medicine is to investigate whether those cultured cells retain tissue regeneration capabilities. To experimentally prove this, our group performed transplantation experiments ${ }^{20)}$. CoIonic epithelial cells of transgenic mice that ubiquitously express EGFP were cultured and infused into the colonic lumen of recipient mice in which colonic epithelial injuries were generated. What we found is that, around 1 week after transplantation, EGFP+ donor-derived cells were incorporated into the denuded regions of recipients' colon as a flat sheet-like lining. At 4 weeks, most of the EGFP+grafts were composed of crypts in which EGFP+ cells extended from the bottommost positions to their tops, indicating that those crypts were entirely replaced by donor-derived cells. Histological analysis revealed that all types of terminally differentiated colonic epithelial cells as well as proliferating cells were present in the donor-derived epithelium (Fig. 1). This clearly indicates that a population of transplanted cells really functioned as stem cells in vivo. Moreover, the success of this stem cell transplantation could also be achieved even with the donor cells that were grown from 
a single Lgr5+ stem cell in culture. The single cell-derived transplanted cells were found to generate multiple crypts in multiple recipient mice, indicating that the stem cells really expand in the preceding process of culture, keeping their stem cell properties unaffected ${ }^{20)}$.

\section{Transplantation of fetal intestinal epithelial progenitor cells into adult colons}

Not only for adult intestinal epithelial cells, the cuttingedge cell culture technology has also been applied for intestinal cells of fetal origin. Fordam et al. reported that fetal SI epithelial progenitors are able to grow as fetal enterospheres (FEnS), which are characterized by distinct proliferative and differentiation potential as compared to the adult SI stem cells ${ }^{21)}$. As high levels of Wnt factors can induce the transition of those fetal cells into adult state, those cells in culture are thought to phenocopy the immature state of intestinal cells in early developmental stages. In collaboration with this group in Copenhagen, we have shown that the FEnS-derived cells can also graft onto adult colons and thereby would be a transplantable source for regenerative medicine. Interestingly, when transplanted onto the adult colon, FEnS-derived cells started to express CA2, a marker protein of colonic epithelium, even though its expression was not detectable prior to the transplantation ${ }^{21)}$. This suggests that fetal SI progenitors have plasticity in regard to their fate and are able to adapt to the new microenvironment (Fig.1).

\section{Transplantation of small intestinal stem cells of adult origin into colons}

Then, how do cultured SI cells of adult origin behave in vivo when they are transplanted back into the body? By extending our transplantation approach, we have recently assessed this ${ }^{22)}$. SI cells were isolated from adult mice and cultured as stem cell-containing organoids as previously described $^{3)}$. They were then collected and instilled into the colonic lumen of wild-type recipient mice in which injuries were generated in advance. We found that transplanted SI cells adhered to the denuded colonic tissue retaining their epithelial phenotype. Interestingly, donor-derived cells did not show obvious expression of CA2, which contrasts to the in vivo behavior of fetal SI epithelial cells ${ }^{21)}$. Moreover, even at later time points, transplanted SI cells were shown to contain all types of terminally differentiated cells and the epithelial stem cells of SI phenotype. In addition, the intestinal villi, typical structures unique only to the SI but

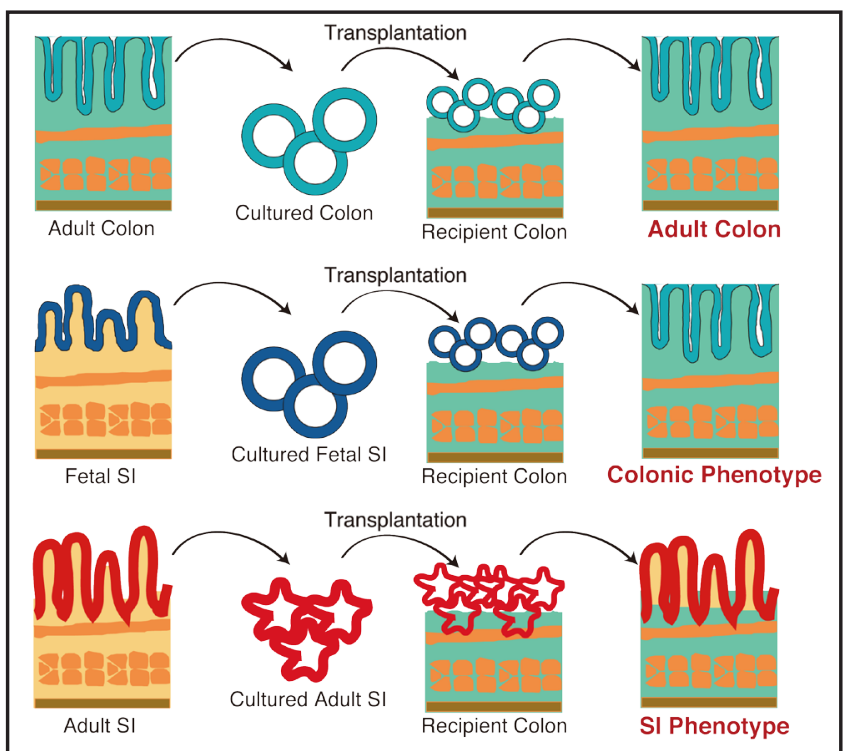

Fig. 1 Transplantation experiments using different cell sources result in different outcome

Schematic representation of the transplantation studies. Cells obtained from the adult colon (top), fetal SI (middle), and adult SI (bottom) were grown in culture and then transplanted into recipient mice in which colonic epithelial injuries were generated. Regenerated epithelia from the cultured adult colonic cells showed colonic phenotype. Fetal SI-derived cells generated epithelial tissues in part adapting to the colonic microenvironment. Transplanted cells originating from the adult SI stayed as SI cells even in the colonic milieu.

not to colon, were clearly visible in some parts of the graft. Together with the presence of functional Paneth cells in the transplanted epithelia, it was shown that cultured adult SI stem cells are able to function as genuine stem cells to reconstitute normal epithelia of SI phenotype ${ }^{22)}$. This study provides proof of principle that cultured SI stem cells could also be a source for cell therapy of intestinal diseases. Moreover, it is further suggested that adult SI cells reconstitute epithelia in a manner different from that of fetal SI progenitor cells, as they maintain their identity along the gastrointestinal tract even after being heterotopically transplanted.

\section{Conclusion}

In this article, I have tried to provide a brief introduction into the current status of intestinal stem cell research, including the experimental evidence that shows feasibility of stem cell based therapy for intestinal epithelial injuries. There are many severe Gl diseases, such as congenital disorders or inflammatory bowel diseases, which affect 
intestinal epithelial cells leaving patients with few treatment options. There are still many questions to be answered before regenerative medicine can become a clinical reality. It is not fully clear whether the grafted tissues are functionally normal in terms of absorption, secretion, endocrine function, immunoregulatory function and so on. Whether or not the genetic/epigenetic information is stably maintained during the culture should be clarified in detail. However, steady progress in this research field would allow us to envisage realistic future situations where GI stem cells would be the reliable source for regenerative medicine in human diseases.

\section{Sources of funding}

None

Conflict of interests

None

\section{References}

1) Barker N, van Es JH, Kuipers J, et al: Identification of stem cells in smallintestine and colon by marker gene Lgr5. Nature. 2007; 449: 1003-1007.

2) Jung $P$, Sato $T$, Merlos-Suarez $A$, et al: Isolation and in vitro expansion of human colonic stem cells. Nat Med. 2011; 17: 1225-1227.

3) Sato T, Vries RG, Snippert HJ, et al: Single Lgr5 stem cells build crypt-villus structures in vitro without a mesenchymal niche. Nature. 2009; 459: 262-265.

4) Ootani A, Li X, Sangiorgi E, et al: Sustained in vitro intestinal epithelial culture within a Wnt-dependent stem cell niche. Nat Med. 2009; 15: 701-706.

5) Sato $T$, Stange DE, Ferrante $M$, et al: Long-term expansion of epithelial organoids from human colon, adenoma, adenocarcinoma, and Barrett's epithelium. Gastroenterology. 2011; 141: 1762-1772.

6) Bjerknes $M$, Cheng $\mathrm{H}$ : Intestinal epithelial stem cells and progenitors. Methods Enzymol 2006; 419: 337-383.

7) Barker $N$, van de Wetering $M$, Clevers $H$ : The intestinal stem cell. Genes Dev. 2008; 22: 1856-1864.

8) Sancho E, Batlle E, Clevers H: Signaling pathways in intestinal development and cancer. Annu Rev Cell Dev Biol. 2004; 20: 695-723.

9) Podolsky DK: Mucosal immunity and inflammation. V.
Innate mechanisms of mucosal defense and repair: the best offense is a good defense. Am J Physiol. 1999; 277: G495-G499.

10) Booth D, Potten CS. Protection against mucosal injury by growth factors and cytokines. J Natl Cancer Inst Monogr. 2001: 16-20.

11) Crosnier C, Stamataki D, Lewis J: Organizing cell renewal in the intestine: stem cells, signals and combinatorial control. Nat Rev Genet. 2006; 7: 349-359.

12) Radtke $F$, Clevers $H$ : Self-renewal and cancer of the gut: two sides of a coin. Science. 2005; 307: 1904-1909.

13) Korinek V, Barker N, Moerer $P$, et al: Depletion of epithelial stem-cell compartments in the small intestine of mice lacking Tcf-4. Nat Genet. 1998; 19: 379-383.

14) Pinto D, Gregorieff $A$, Begthel $H$, Clevers $H$ : Canonical Wnt signals are essential for homeostasis of the intestinal epithelium. Genes Dev. 2003; 17: 1709-1713.

15) Kuhnert F, Davis CR, Wang HT, et al: Essential requirement for Wnt signaling in proliferation of adult small intestine and colon revealed by adenoviral expression of Dickkopf-1. Proc Natl Acad Sci U S A. 2004; 101: 266-271.

16) Sangiorgi $E$, Capecchi MR: Bmi1 is expressed in vivo in intestinal stem cells. Nat Genet. 2008; 40: 915-920.

17) Tian $H$, Biehs $B$, Warming $S$, et al: A reserve stem cell population in small intestine renders Lgr5-positive cells dispensable. Nature. 2011; 478: 255-259.

18) Barker N. Adult intestinal stem cells: critical drivers of epithelial homeostasis and regeneration. Nat Rev Mol Cell Biol. 2014; 15: 19-33.

19) Barker N, Huch M, Kujala $P$, et al: Lgr5(+ve) stem cells drive self-renewal in the stomach and build long-lived gastric units in vitro. Cell Stem Cell. 2010; 6: 25-36.

20) Yui S, Nakamura T, Sato T, et al: Functional engraftment of colon epithelium expanded in vitro from a single adult Lgr5(+) stem cell. Nat Med. 2012; 18: 618-623.

21) Fordham RP, Yui S, Hannan NR, et al: Transplantation of expanded fetal intestinal progenitors contributes to colon regeneration after injury. Cell Stem Cell. 2013; 13: 734-744.

22) Fukuda M, Mizutani T, Mochizuki W, et al: Small intestinal stem cell identity is maintained with functional Paneth cells in heterotopically grafted epithelium onto the colon. Genes Dev. 2014; 28: 1752-1757. 